\title{
Analysing the role of Saraswatarishta in the treatment of neurological disorders based on network pharmacology
}

\author{
Vrinda Jethalia, Sanjana Varada Hasyagar, Kasturi Bhamidipati and Jhinuk Chatterjee * \\ Department of Biotechnology, PES University, Karnataka, India. \\ * Correspondence: jhinukchatterjee@pes.edu; Tel.: +91-802-672-6672 (345) \\ Received: 7 July 2021; Accepted: 28 August 2021; Published: 18 September 2021 \\ Edited by: Indranath Chatterjee (Tongmyong University, Republic of Korea) \\ Reviewed by: Prashanthi K (MS Ramaiah University of Applied Sciences, India); \\ MS Zobaer (The University of Texas Health Science Center at Houston (UTHealth), USA); \\ Alina Arulsamy (Monash University Malaysia, Malaysia) \\ https://doi.org/10.31117/neuroscirn.v3i5.106
}

\begin{abstract}
Ayurvedic medications originated centuries ago and are still prevalent today. Saraswatarishta (SWRT) is a well-known ayurvedic formulation that is often prescribed to control the manifestations of neurological illnesses and disorders such as slurred speech, anxiety, Parkinson's disease (PD) and Alzheimer's disease(AD). However, scientific research on its mode of action has not been studied extensively. Therefore, this study employs network pharmacology to understand better the neuroprotective role of Saraswatarishta (SWRT) in neurological disorders. Out of the 18 ingredients in SWRT, five were considered in this study due to their elevated therapeutic action in neurological disorders. Further, nine active phytoconstituents were chosen from the five selected ingredients. The gene targets of the active phytoconstituents were screened and selected using STITCH, SwissTargetPrediction and ChEMBL. Protein-Protein interaction and Gene Ontology (GO) enrichment analysis were carried out using STRING and g:Profiler, respectively. Cytoscape 3.7.2 was used to create three networks-the compound-target, the target-disease and the compound-targetdisease network. Molinspiration and admetSAR2.0 were used to obtain the bioactivity scores and the bloodbrain barrier (BBB) probability scores. The three networks indicated that all nine phytoconstituents were linked to the gene targets that encode proteins involved in the pathways of 10 major neurological disorders. This includes Parkinson's disease (PD), Alzheimer's disease (AD), dementia, Huntington disease, epilepsy, schizophrenia, spinocerebellar ataxia, amyotrophic lateral sclerosis (ALS), multiple sclerosis and attention deficit hyperactivity disorder (ADHD). The gene targets were expressed significantly in various central nervous system regions such as the cerebral cortex, cerebellum and amygdala. The bioactivity scores of the phytoconstituents were in the active range along with high $B B B$ probability scores, indicating that the phytoconstituents can potentially cross the BBB and impart therapeutic effects.
\end{abstract}

Keywords: SWRT; PPI; phytoconstituents; Ayurveda; network pharmacology; Saraswatarishta;

(C2021 by Jethalia et al. for use and distribution according to the Creative Commons Attribution (CC BY-NC 4.0) license (https://creativecommons.org/licenses/by-nc/4.0/), which permits unrestricted non-commercial use, distribution, and reproduction in any medium, provided the original author and source are credited.

\subsection{INTRODUCTION}

Ayurveda, which translates to 'knowledge of life' in Sanskrit, is an alternative medicine system that originated several thousand years ago in India. It places great emphasis on the prevention of diseases by encouraging the maintenance of health by paying close attention to the balance in one's life, their rightful thinking, their diet and lifestyle, and the use of herbs (Gadgil, 2010). However, scientific research on ayurvedic medication is not prominent. Therefore, two key questions remain - how it works and why it works. These questions need to be answered because when 
phytomedicines in the ayurvedic medicine system are provided with scientific backing and clinical trials, they will receive further recognition and acceptance for use.

Among the various formulations present in the ayurvedic system, Saraswatharishta (SWRT) is one such formulation that has shown proven effects in the treatment and management of neurological illnesses and disorders. An in vitro study on SWRT's effect showed that it enhanced the memory of mice treated with Scopolamine (Prabhu et al., 2020), while performing better than Piracetam, a commercial drug for neuro-protection. In another study, when Albino mice were pre-treated with SWRT for two weeks, they gained protection against the memory and learning impairment effects of Diazepam (Rajopadhye \& Sahasrabudhe, 2020). The above-mentioned examples of experimental research on SWRT's effect in mice showed that it imparts neuroprotective properties and that it is worthwhile to begin exploring its mechanism of action.

From an in silico standpoint, this study aimed to investigate the mechanism of action and pharmacological properties of the active phytochemicals in SWRT that may play a significant role in alleviating neurological disorders. Network pharmacology, a key concept concerning the multitarget nature (Chandran et al., 2015; Kumar et al., 2018) was implemented in this study. Network pharmacology is shifting the current "one drug-one target" paradigm since the latter fails to consider the multifactorial nature of diseases (Chandran et al., 2015). For example, current research in Alzheimer's disease (AD) adopts a more viable solution that simultaneously modulates more than one target in the neurodegenerative cascade (Kumar et al., 2018). A "7-mehtoxytacrine (7-MEOTA)" derivative was developed based on the multi-target directed ligand that has raised new hope in the treatment of AD (Gazova et al., 2016).

The SWRT formulation consists of 18 ingredients, which are Bacopa monnieri, Asparagus racemosus, Pueraria tuberosa, Terminalia chebula, Zinziber officinalis, Anethum sowa, Operculina ipomoea, Piper longum, Syzygium aromaticum, Acorus calamus, Saussurea lappa, Withania somnifera, Terminalia belerica, Tinospora cordifolia, Elettaria cardamomum, Embelia ribes, Cinamomum zelonica and pure gold (Parekar et al., 2014). Studies have shown that out of these 18 ingredients, Bacopa monnieri (Ramasamy et al., 2015; Jeyasri et al., 2020; Chaudhari et al., 2017), Withania somnifera (Kumar et al., 2015; Saroya \& Singh, 2018),
Syzygium aromaticum (Batiha et al., 2020), Elettaria cardamomum (Abu-Taweel, 2018) and Zingiber officinale (Mao et al., 2019; Liu et al., 2019) may play a more significant role in easing neurological symptoms as compared to the others. Therefore, by employing the concept of network pharmacology, this study focuses on understanding the role of the active phytoconstituents in the five aforementioned ingredients of SWRT. This, in turn, will throw some light on the neuroprotective mode of action that SWRT imparts.

Nine active phytoconstituents from the five ingredients were analysed by obtaining their predicted gene targets, performing protein-protein interaction and GO enrichment analysis, disease analysis, creating networks, obtaining bioactivity scores and blood-brain barrier probability scores and comparison with commercial drugs. This methodology provided new insights into SWRT's effect on neurological disorders. Many common proteins that play a role in the pathogenesis of neurological disorders emerged in our networks and had connections with one or more of the nine active phytoconstituents. The predicted bioactivity and BBB probability scores of these selected ingredients were on par or better than the already existing commercial drugs. The flowchart of the complete study design was illustrated in Figure 1.

\subsection{MATERIALS AND METHODS}

\subsection{Screening of the SWRT ingredients and their respective phytoconstituents}

The 18 ingredients of SWRT were reviewed individually with the help of existing literature. After studying the 18 ingredients, five were considered in this study, as they were seen to play a more significant role in mitigating neurological disorders. The phytoconstituents of the five ingredients were further studied using literature sources to identify the phytoconstituents that are active in imparting neuroprotective effects.

\subsection{Target retrieval and validation}

The canonical smiles ID, molecular formula and molecular weight of the identified phytoconstituents were retrieved from PubChem (Kim et al., 2021). The human biological targets for each of the phytoconstituents were obtained from SwissTargetPrediction (Daina et al., 2019), ChEMBL (Mendez et al., 2019) and STITCH (Kuhn et al., 2008). To gather information about the targets, the gene names were entered individually in GeneCards (Safran et al., 2002), and the results were obtained from the 'pathway' and 'expression' sections. DisGeNET (Piñero et al., 2017) 


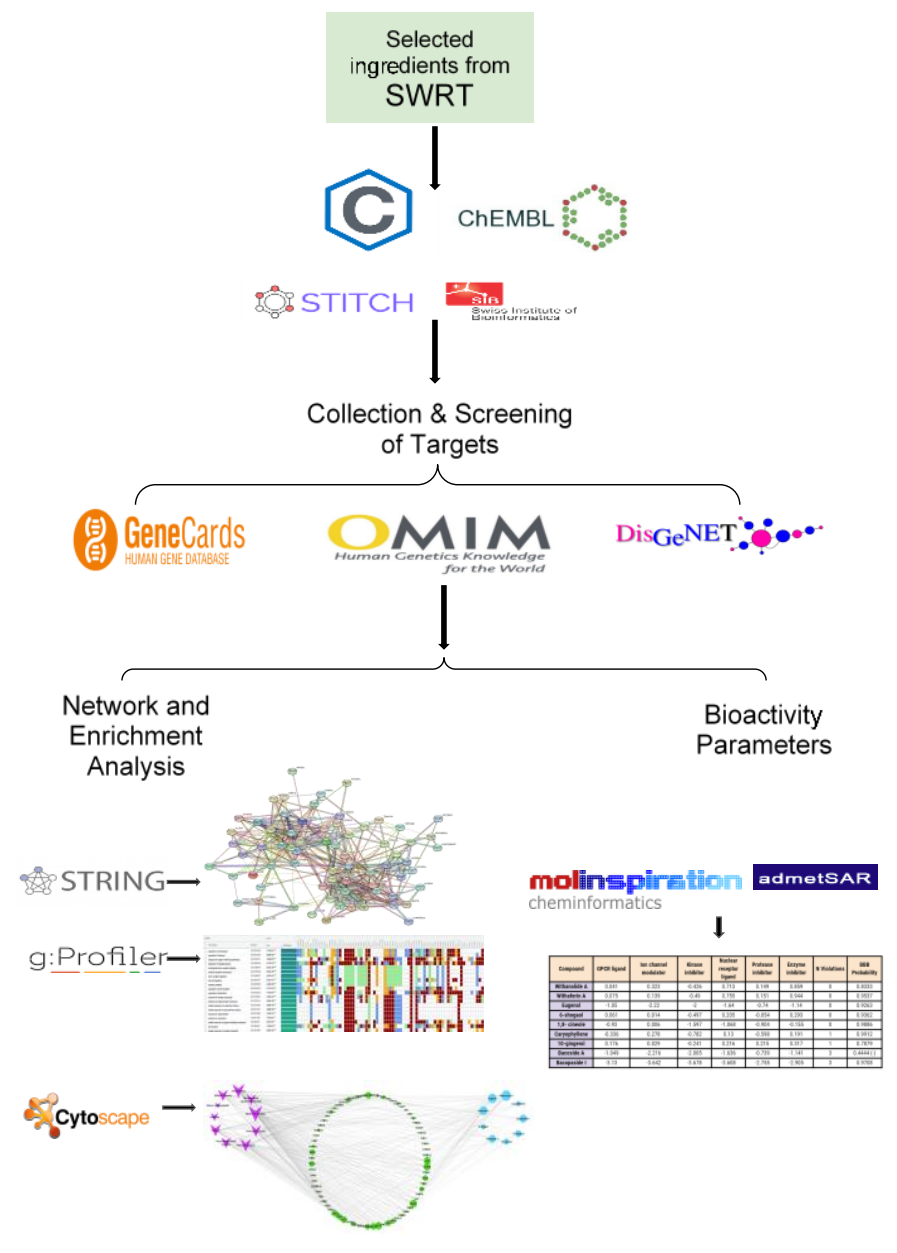

Figure 1: Flow chart representing the study design.

and OMIM (Online Mendelian Inheritance in Man) (Hamosh et al., 2005) were used to identify the diseases in which the selected targets played an active role.

\subsection{Protein-protein-interaction (PPI), enrichment and} network analysis

The targets were imported to STRING (Szklarczyk et al., 2019), where the protein-protein interaction (PPI) network was constructed, and PPI enrichment analysis was performed. The default parameters - organisms set to Homo Sapiens and required score set to medium confidence (0.004), were used to create the PPI network. The targets were also subjected to GO (Gene Ontology) enrichment on g:Profiler (Raudvere et al., 2019). The input was the official gene symbols, the organism option set to Homo Sapiens and the advanced options remained as default. Under data sources, only GO biological process, GO molecular function, GO cellular component, and KEGG (Kyoto Encyclopedia of Genes and Genomes) were selected.

Cytoscape 3.7.2 (Shannon et al., 2003) was used to construct and visualise the Compound-Target network, Disease-Target network and Compound-Target-Disease network. The interactions were considered directed, which implies that the connection points from one node to another in one direction. The nodes and edges were given the "Low values to small sizes" representation, keeping EdgeCount as the focal point in the network analysis. This means that the node size will be small if there are a lower number of edges emerging from/entering it.

\subsection{Bioactive scores and their comparison with commercial drugs}

The canonical smiles ID of the compounds were uploaded on Molinspiration (http://www.molinspiration.com) to retrieve the predicted values of molecular properties like kinase inhibitor activity, G-protein-coupled receptor ligand activity, nuclear receptors, protease inhibitor activity and the number of violations. The admetSAR 2.0 (Yang et al., 2019) was used to predict whether the phytoconstituents could cross the blood-brain barrier (BBB) by generating a probability score using its classification-based BBB model.

\subsection{RESULTS}

\subsection{SWRT phytoconstituents}

The five ingredients found in the SWRT formulation that were considered in this study include Bacopa monnieri, Withania somnifera, Syzygium aromaticum, Elettaria cardamomum and Zingiber officinale. From these, a total of 9 phytoconstituents that play a major role in mitigating neurological disorders were selected by studying the existing neuropharmacology literature.

The selected phytoconstituents from Withania somnifera were Withanolide A (Zhu et al., 2020, Baitharu et al., 2014) and Withaferin A (Ram et al., 2021); from Bacopa monnieri were Bacoside A (Jeyasri et al., 2020) and Bacopaside I (Aguiar \& Borowski, 2013); from Syzygium aromaticum, were Eugenol and Caryophyllene (Galal \& Abdellatief, 2015); from Zingiber officinale were 6-shogaol ( $\mathrm{Ha}$ et al., 2012) and 10gingerol (Mao et al., 2019; Liu et al., 2019); and lastly from Elettaria cardamomum, it was 1,8 cineole (Zheng et al., 2019).

\subsection{Selection of targets and validation}

Initially, a total of 411 targets were generated as results from SwissTargetPrediction, ChEMBL and STITCH. The 411 targets were narrowed down to 96 targets as a result of the preliminary screening, which was based on whether the gene targets were traditionally CNS targets or not. The 96 targets were imported into GeneCards, where its 'pathway' and 'expression' sections were used 
to analyse the gene targets further. Various pathway tools linked to GeneCards, such as Reactome, KEGG Pathway, BioSystems and PharmGKB, displayed the pathways in which the imported targets were present. It was observed that the chosen targets were mostly present in the central nervous system (CNS) related pathways, and they were significantly expressed in the brain, cortex, cerebellum, retina, spinal cord or the tibial nerve. A total of 10 major neurological disorders, which were chosen based on their occurrence within our imported dataset on DisGeNET, were considered in this study. The ten neurological disorders considered were Parkinson's disease (PD), Alzheimer's disease (AD), dementia, Huntington disease, epilepsy, schizophrenia, spinocerebellar ataxia, amyotrophic lateral sclerosis (ALS), multiple sclerosis and attention deficit hyperactivity disorder (ADHD). Based on the results obtained from DisGeNET and OMIM, it was shown that most of the gene targets encoded proteins that were involved in at least one or more of these diseases. Those targets that were not present in the CNS related pathways or were not expressed in either the brain, cortex, cerebellum, retina, spinal cord or the tibial nerve were rejected, resulting in a final and accurate collection of 71 gene targets. These validated 71 targets were used in the rest of the study.

\subsection{Protein-protein interaction (PPI) and GO analysis}

The PPI network obtained from STRING had 71 nodes and 430 edges. Most of the interactions were either known interactions (either curated from databases, experimentally determined or both) or predicted interactions (gene neighbourhood, gene fusions and gene co-occurrence). The interaction enrichment indicated that the proteins were at least partially biologically connected as a group.

In the GO enrichment analysis performed on g:Profiler, biological process, molecular function and cellular component were considered, which consisted of 550, 95 and 64 GO enriched terms, respectively. The KEGG pathway enrichment produced 79 enriched terms. The top 20 enriched terms for biological process, molecular function, cellular component and KEGG pathway were considered, which were sorted based on the lowest $p$ adjusted value. The lower the adjusted $p$-value, the higher the significance of the term.

The GO enrichment analysis revealed that most of the GO terms were neurologically related. For example, some of the enriched molecular functions and biological processes were neurotransmitter receptor activity, serotonin receptor activity, ion transmembrane transporter activity, regulation of ion transport and synaptic signalling. In the case of cellular components, the enriched terms were neuron projection, synapse, and dendrite, to name a few. KEGG pathway enrichment results included calcium signalling pathway, cAMP signalling pathway, Alzheimer's disease, and "Pathways of neurodegeneration - multiple diseases", a generalised KEGG Pathway term.

\subsection{Network Analysis}

\subsubsection{Compound-Target Network Analysis}

As illustrated in Figure 2, the compound-target network represents the multi-target approach, which is commonly observed in ethnopharmacology. In addition, multiple phytoconstituents interacted with a particular target giving rise to certain synergistic modes of action in treating neurological disorders by either inhibiting or modulating the gene products. SLC9A1 shared connections with three phytoconstituents- Bacopaside I, Bacoside A and Eugenol. This indicated that these three phytoconstituents might potentially inhibit SLC9A1, thereby useful in treating the related neurological disorders. Similar to SLC9A1, ALOX5 also shared connections with three phytoconstituentsEugenol, 6-shogaol and 10-gingerol. GSK3B, PTGS2 and PRKCD shared connections with Withaferin $A$, and Withanolide A. CASP8 shared connections with Bacoside A and Withanolide A while TRPV1 had connections with 1,8- cineole, Caryophyllene and Eugenol.

\subsubsection{Target-Disease Network Analysis}

The target-disease network displayed in Figure $\mathbf{3}$ revealed that the targets shared connections with the ten neurological disorders emphasised in this study. These targets were connected to one or more diseases, which implied that they were involved in the pathogenesis of the disease. For example, ALOX5 was connected to PD, AD, dementia, Huntington disease, epilepsy and multiple sclerosis. Similarly, GSK3B, CASP8 and PTGS2 shared common connections with PD, AD, Huntington disease, and epilepsy. SLC9A1 was connected to Huntington disease and epilepsy. PRKCD had connections with PD, AD, epilepsy, schizophrenia and ADHD, while TRPV1 shared its connections with AD, $P D$, epilepsy, schizophrenia and multiple sclerosis.

\subsubsection{Compound-Target-Disease Network Analysis}

The compound-target-disease network in Figure 4 provided a collective representation of the targets involved in neurological disorders, which may be controlled by the phytoconstituents it shared a 


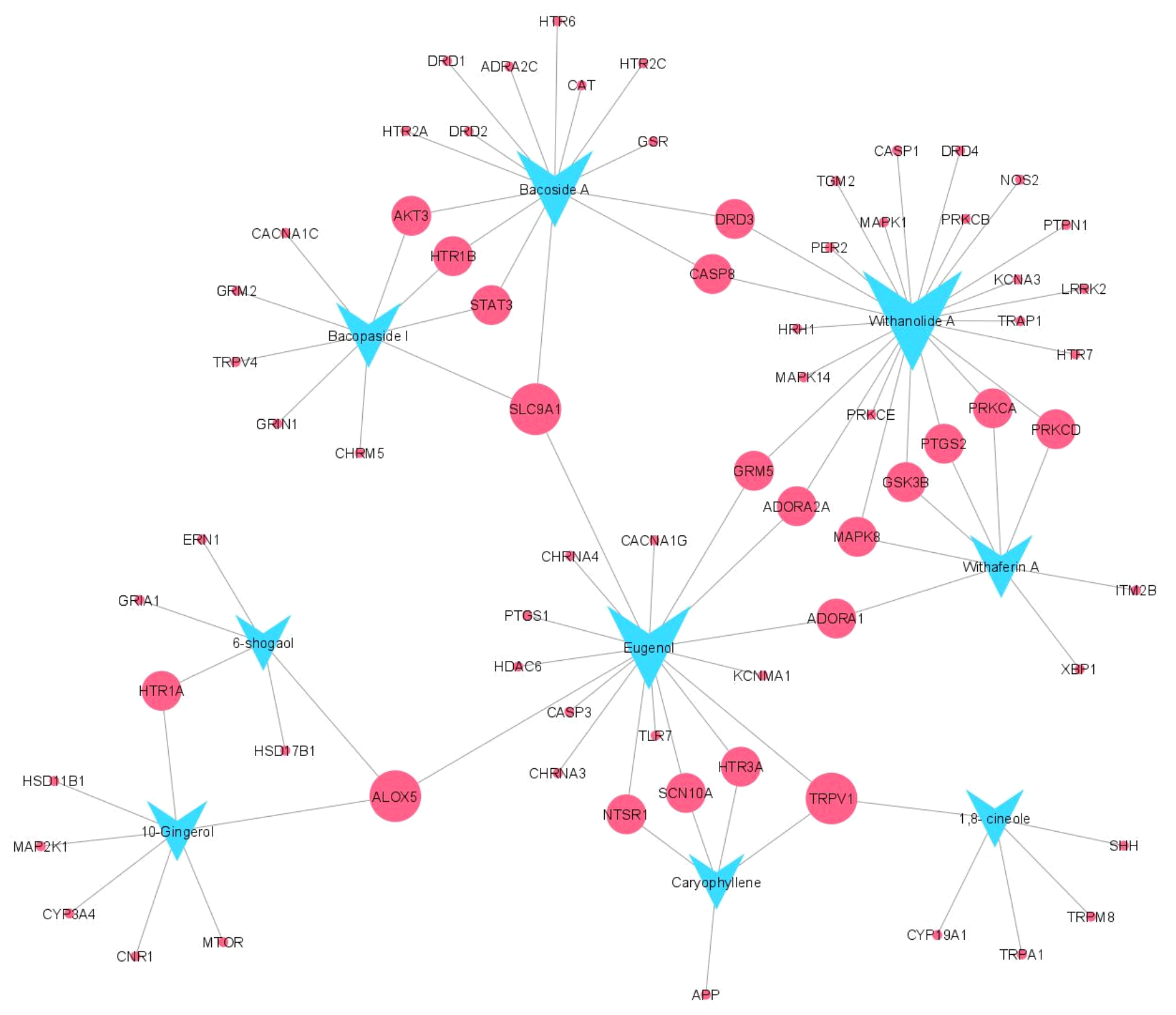

Figure 2: Compound-Target Network. This network consists of 80 nodes and 94 edges. Circles represent targets and V-shaped arrows represent phytoconstituents. A large node size indicates more number of edges emerging that node and vice versa.

connection with. For instance, it was shown that the GSK3B was involved in the pathogenesis of $A D, P D$, epilepsy, dementia, ADHD and schizophrenia. It also shared connections with Withanolide $A$ and Withaferin $A$, indicating that these phytoconstituents may also control their function, thereby treating their related disease. In this manner, ALOX5 was connected to PD, $A D$, dementia, Huntington disease, epilepsy and multiple sclerosis. It also shared connections with three phytoconstituents: Eugenol, 6-shogaol and 10-gingerol. GSK3B, PTGS2 and PRKCD had connections with PD, AD, dementia, Huntington disease, epilepsy, schizophrenia. They shared connections with Withaferin $A$ and Withanolide $A$, indicating the potential role of these phytoconstituents in treating related neurological disorders.

\subsection{Bioactivity parameters}

The phytoconstituents were compared to commercial drugs based on their bioactivity parameters (Table 1 and Table 2). A value between 0 to 5 indicated that the compound was highly active, 0 to -5 meant the compound was moderately active, and any value below -5 suggested that the compound was inactive. Therefore, the results gathered from the Molinspiration tool revealed that all the phytoconstituents had a bioactivity value in the active range. In most cases, the phytoconstituents displayed a similar or better score when compared to the commercial drugs prescribed for neurological disorders. For instance, Withanolide A has a higher value for G-protein coupled receptor (GPCR) ligand, ion channel modulator, nuclear receptor ligand, protease inhibitor, and enzyme inhibition compared to 


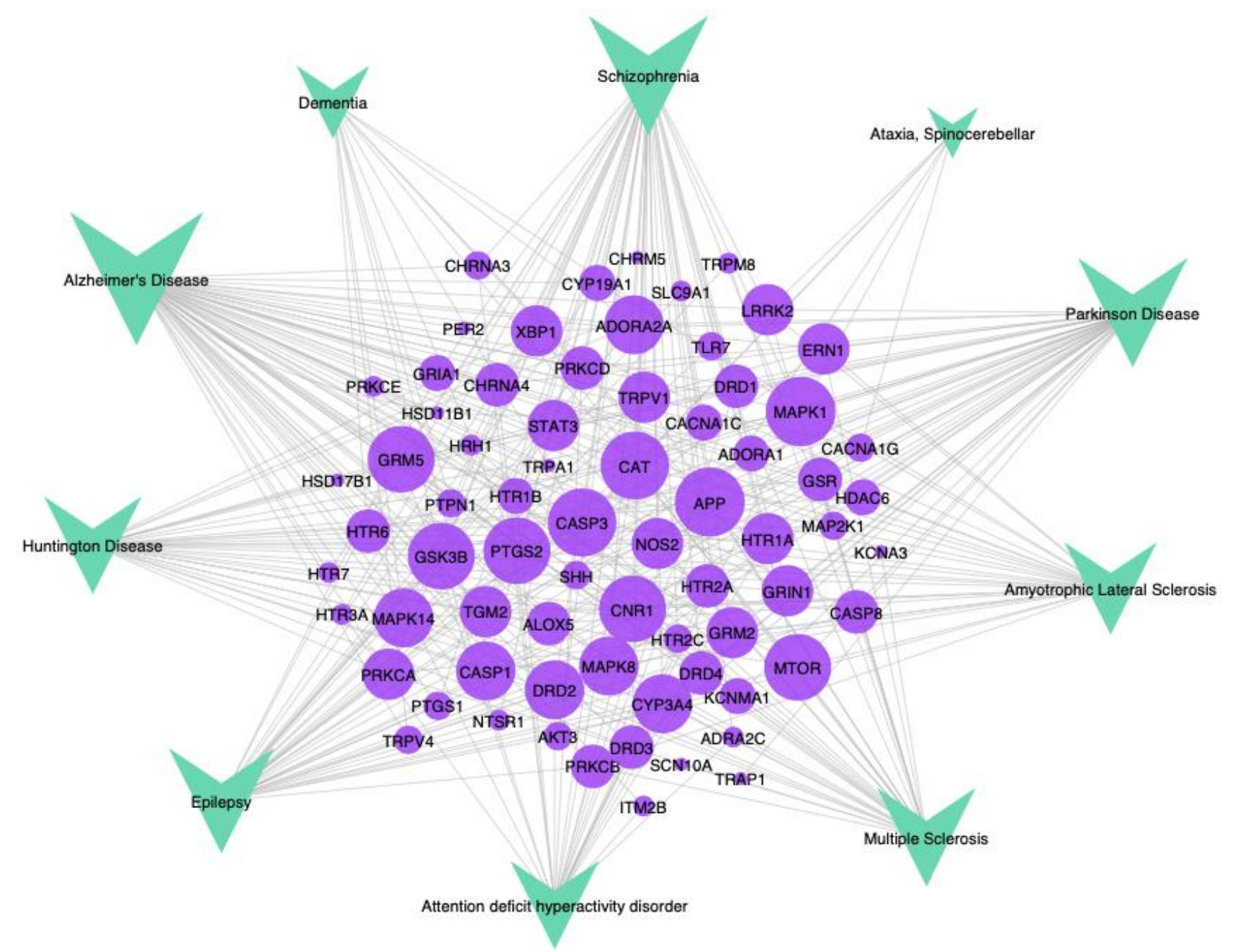

Figure 3: Target-Disease Network. This network comprises 81 nodes and 325 edges. Circles represent targets, and V-shaped arrows represent the neurological disorder. Large node size indicates more number of edges emerging that node and vice versa.

Donepezil, which is a commercial medication used to treat Alzheimer's disease.

The positive values of the phytoconstituents concerning the blood-brain barrier parameter obtained from admetSAR2.0 implied that these phytoconstituents could cross the BBB and impart therapeutic effects on neurological disorders.

\subsection{DISCUSSION}

The development of effective medicines for the treatment of neurological disorders has been challenging. Therefore, research and development of alternative forms of medicines should be accelerated as they may help alleviate symptoms of these neurological disorders. Ayurveda has been a widely used alternative form of medication for several thousand years. However, there is a lack of clear understanding of its mechanism of therapeutic action, which ultimately served as the motivation for this study.

SWRT is an ayurvedic formulation prescribed for many diseases such as autoimmune diseases, skin disorders, gynaecological problems and neurological disorders (Shastri, 2002; Prabhu et al., 2019). In this study, novel research was conducted to understand Saraswatharishta's role in treating neurological disorders using network pharmacology. To our knowledge, there isn't previously published literature on analysing this formulation from the gene level. The "one disease-one drug-one target" approach tends to neglect the influence of the complex association of various targets in diseases (Kumar et al., 2018). Whereas in network pharmacology, the problem is assessed from a larger perspective, and it also enables factoring in the synergistic mechanisms of compounds which is often excluded in the previous approach 


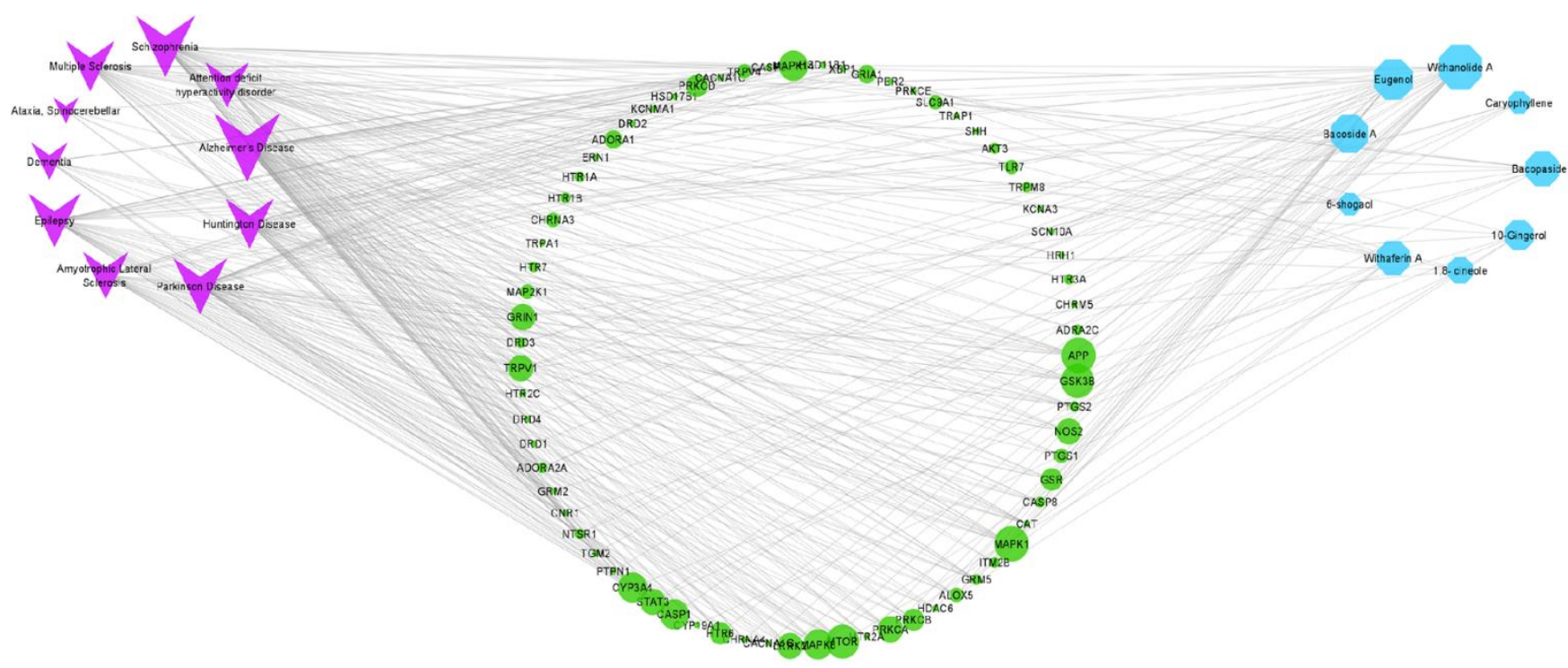

Figure 4: Compound-Target-Disease Network. This network comprises 90 nodes and 418 edges. Circles represent targets, and V-shaped arrows represent neurological disorders, and hexagons represent phytoconstituents. Large node size indicates more number of edges emerging that node and vice versa.

Table 1: Bioactivity parameters and BBB probability of the phytoconstituents

\begin{tabular}{lcccccccc}
\hline \multicolumn{1}{c}{ Compound } & $\begin{array}{c}\text { GPCR } \\
\text { ligand }\end{array}$ & $\begin{array}{c}\text { Ion channel } \\
\text { modulator }\end{array}$ & $\begin{array}{c}\text { Kinase } \\
\text { inhibitor }\end{array}$ & $\begin{array}{c}\text { Nuclear } \\
\text { receptor ligand }\end{array}$ & $\begin{array}{c}\text { Protease } \\
\text { inhibitor }\end{array}$ & $\begin{array}{c}\text { Enzyme } \\
\text { inhibitor }\end{array}$ & $\begin{array}{c}\text { N } \\
\text { Violations }\end{array}$ & $\begin{array}{c}\text { BBB } \\
\text { Probability }\end{array}$ \\
\hline Withanolide A & 0.041 & 0.323 & -0.426 & 0.713 & 0.149 & 0.859 & 0 & 0.8333 \\
\hline Withaferin A & 0.075 & 0.139 & -0.49 & 0.755 & 0.151 & 0.944 & 0 & 0.9537 \\
\hline Eugenol & -1.05 & -2.22 & -2 & -1.64 & -0.74 & -1.14 & 0 & 0.9263 \\
\hline 6-shogaol & 0.061 & 0.014 & -0.497 & 0.205 & -0.054 & 0.293 & 0 & 0.9362 \\
\hline 1,8- cineole & -0.93 & 0.006 & -1.597 & -1.068 & -0.904 & -0.155 & 0 & 0.9886 \\
\hline Caryophyllene & -0.336 & 0.278 & -0.782 & 0.13 & -0.598 & 0.191 & 1 & 0.9912 \\
\hline 10-gingerol & 0.176 & 0.029 & -0.241 & 0.216 & 0.215 & 0.317 & 1 & 0.7879 \\
\hline Bacoside A & -1.049 & -2.216 & -2.005 & -1.636 & -0.739 & -1.141 & 3 & $0.4444(-)$ \\
\hline Bacopaside I & -3.13 & -3.642 & -3.678 & -3.608 & -2.755 & -2.905 & 3 & 0.9708 \\
\hline
\end{tabular}

Notes: The table represents the drug-likeness score towards GPCR ligands, ion channel modulators, kinase inhibitors, nuclear receptor ligands, protease inhibitors and other enzyme targets of phytoconstituents. The number of violations in following Lipinski's rule of 5 and the molecule's probability to cross the blood-brain barrier. Highly active compound (0 to 5 ), Moderately active compound ( 0 to -5 ) and inactive compound (below -5 )

(Chandran et al., 2015). Hence, in the present study, using network pharmacology, the role of the active phytoconstituents derived from the five selected ingredients of SWRT that were closely related in the treatment of neurological disorders were analysed. There have been published network pharmacology research on Withania somnifera and Bacopa monnieri's therapeutic action on neurological disorders (Duyu et al., 2021; Jeyasri et al., 2020). Since they are a part of the ingredients of Saraswatharishta formulation, a brief comparison of our findings with these two published pieces of research are mentioned further in the discussion.

The gene targets were screened using databases such as SwissTarget Prediction Server, ChEMBL and STITCH. Interestingly, most of the targets showed significant expression levels in the CNS on the GeneCards database, thereby validating our selection of targets. A 
Table 2: Bioactivity parameters and BBB probability of commercial drugs for neurodegenerative disorders

\begin{tabular}{|c|c|c|c|c|c|c|c|c|}
\hline Drugs & $\begin{array}{l}\text { GPCR } \\
\text { ligand }\end{array}$ & $\begin{array}{l}\text { Ion channel } \\
\text { modulator }\end{array}$ & $\begin{array}{c}\text { Kinase } \\
\text { inhibitor }\end{array}$ & $\begin{array}{l}\text { Nuclear } \\
\text { receptor ligand }\end{array}$ & $\begin{array}{l}\text { Protease } \\
\text { inhibitor }\end{array}$ & $\begin{array}{l}\text { Enzyme } \\
\text { inhibitor }\end{array}$ & $\begin{array}{c}\mathbf{N} \\
\text { Violations }\end{array}$ & $\begin{array}{c}\text { BBB } \\
\text { Probability }\end{array}$ \\
\hline Primidone & -0.064 & -0.076 & -0.582 & -0.639 & -0.376 & -0.064 & 0 & 1 \\
\hline Deutetrabenazine & 0.004 & -0.185 & -0.666 & -0.417 & -0.142 & -0.254 & 0 & 0.9789 \\
\hline Donepezil & 0.224 & -0.139 & -0.163 & 0.028 & 0.034 & 0.247 & 0 & 1 \\
\hline Apomorphine & 0.574 & 0.378 & -0.077 & -0.13 & -0.037 & 0.336 & 0 & 1 \\
\hline Carbamazepine & 0.015 & 0.354 & -0.102 & -0.541 & -0.319 & 0.244 & 0 & 1 \\
\hline Fingolimod & 0.416 & 0.089 & 0.076 & 0.021 & 0.286 & 0.14 & 0 & 0.8199 \\
\hline Clozapine & 0.334 & 0.342 & 0.48 & -0.287 & -0.139 & 0.173 & 0 & 1 \\
\hline Bromperidol & 0.21 & 0.08 & -0.14 & -0.07 & -0.11 & 0.1 & 0 & 1 \\
\hline Atomoxetine & 0.2 & -0.15 & -0.07 & 0.14 & -0.07 & 0.13 & 0 & 1 \\
\hline Riluzole & -0.522 & -0.035 & -0.31 & -0.801 & -0.604 & -0.21 & 0 & 0.9789 \\
\hline Memantine & -0.284 & 0.115 & -1.088 & -1.07 & -0.603 & -0.473 & 0 & 0.9968 \\
\hline
\end{tabular}

Note: The table represents the drug-likeness score towards GPCR ligands, ion channel modulators, kinase inhibitors, nuclear receptor ligands, protease inhibitors and other enzyme targets of commercially available drugs, the number of violations in following Lipinski's rule of 5 and the molecule's probability to cross the blood-brain barrier. Highly active compound (0 to 5$)$, Moderately active compound ( 0 to -5 ) and inactive compound (below -5 ).

total of 71 targets were selected post-screening, which was further used for network pharmacology studies.

According to the observations from GO enrichment analysis, the enriched molecular functions and biological processes were neurotransmitter receptor activity, serotonin receptor activity, ion transmembrane transporter activity, regulation of ion transport and synaptic signalling. For cellular components, the enriched terms were neuron projection, synapse, and dendrite. KEGG pathway enrichment results included calcium signalling pathway, cAMP signalling pathway, Alzheimer's disease and pathways of neurodegeneration in multiple diseases.

From the Compound-Target network, it can be seen that a single phytoconstituent had connections with multiple targets. For example, the network showed that Eugenol has connections with ALOX5, SLC9A1, GRM5, and NTSR1, to name a few. Research has shown that ALOX5 was involved in the formation of beta-amyloid. 5-LO (encoded by ALOX5) directly activated CAMP-response element-binding protein (CREB) which increased the transcription of proteins in the gamma-secretase complex (Chu \& Pratico, 2011). Studies performed on human neuroblastoma cells transfected with an Alzheimer's disease-associated mutation in the APP gene showed that inhibition of the 5-LO or gene disruption of ALOX5 significantly decreased betaamyloid production and gamma-secretase levels (Chu \& Pratico, 2011). Another study showed that spinocerebellar ataxia in 2 patients was linked to a homozygous mutation in the SLC9A1 gene (Iwama et al., 2018). SLC9A1, ALOX5 shared connections with Eugenol in the network. So, Eugenol can play a therapeutic role and reduce the chances of neurological disorders by acting upon these targets. This applies to all the compounds in the network that have connections with various targets involved in the development of neurological disorders.

One of the reasons for the onset of neurodegenerative diseases is excessive neuronal apoptosis (Gorman 2008). Active caspase-8 gets expressed within reactive microglia in the ventral mesencephalon and frontal cortex of individuals with PD and AD (Burguillos et al., 2011). The authors showed that the orderly activation of caspase-8 regulated microglia activation through a PRKCD-dependent pathway. In the case where caspase8 is inhibited, microglia activation gets hindered, resulting in reduced neurotoxicity. Caspase- 8 was also seen to play an essential role in Huntington related neurodegenerative diseases (Sánchez et al., 1999). In another study, it was seen that there was an elevated expression of PRKCD in Alzheimer's disease (Du et al., 2018). Studies showed a marked upregulation of PKC $\delta$ in the microglia of the ventral midbrain region of PD patients compared to controls. Inhibition of PKC $\delta$ activation was suggested as a novel therapeutic strategy in PD treatment (Gordon et al., 2016). 
In the networks of our study, it was seen that CASP8 shared a connection with Withanolide $A$ and Bacoside $A$ and PRKCD shared connections with Withanolide $A$ and Withaferin $A$. These connections can act as leads for further studies which can state that these phytoconstituents may have a significant inhibitory role. Samad et al. (2001) in their research, showed the involvement of PTGS2 in central nervous system (CNS) responses by finding a widespread induction of PTGS2 expression in spinal cord neurons and other regions of the CNS, elevating prostaglandin E2 (PGE2) levels in the cerebrospinal fluid. In their study on rats, it was seen that intraspinal administration of a suitable inhibitor decreased inflammation-induced PGE2 levels. COX-2 (encoded by PTGS2) overexpression was found to be associated with neurotoxicity in acute conditions. The role of COX-2 as a potential therapeutic target for developing efficacious antiepileptic treatment was analysed (Rawat et al., 2020).

$A B$ production, tau phosphorylation was found to be regulated by GSK3 $\beta$. GSK3 $\beta$ inhibition can facilitate new therapeutic avenues for treating neurodegenerative diseases (Lei et al., 2011; Duda et al., 2018). The networks in our study indicated that Withanolide $A$ and Withaferin A shared connections with GSK3B, suggesting their potential inhibitory role. Growing evidence has suggested that TRPV1 can mediate various pathways from glial reactivity and cytokine release to synaptic transmission and plasticity. Authors have highlighted the increasing importance of TRPV1 as a regulator of CNS function (Ho et al., 2012). It has been noticed that the expression of TRPV1, another target in our network, was elevated in the cortex and hippocampus of patients with temporal lobe epilepsy. TRPV1 agonists inhibited the development of seizures by inhibiting TRPV1 (Storozhuk et al., 2019). The networks also illustrated how some targets shared more than one connection with phytoconstituents and diseases. It was seen that ALOX5 shared connections with Eugenol, 6-Shogaol and 10-Gingerol. Similarly, SLC9A1 had a total of 3 connections with Eugenol, Bacoside $A$ and Bacopaside I. This phenomenon was noticed within many targets in the network, providing a concrete lead towards the synergistic mode of action imparted by natural compounds.

All nine phytoconstituents have been reported to show neuroprotective effects in in vitro and in vivo model systems. For example, neuroprotective effects of bacosides were widely studied by in vivo and in vitro models (Abdul Manap et al., 2019). Authors have examined the beneficial effects of Withanolide $A$ involved in neuroprotection and anti-inflammatory effects in a mouse model of pilocarpine-induced status epilepticus (Zhu et al., 2020). The neuroprotective nature of eugenol against toxic amyloids was reported using both in vitro and cellular model systems (Dubey et al., 2017). Results indicated that $\beta$-Caryophyllene had multiple neuroprotective mechanisms in a murine model, and it was suggested that it might be a potential treatment for PD (Viveros-Paredes et al., 2017). The fresh ginger extract exhibited a significant antineuroinflammatory capacity, which was primarily due to 10-gingerol (Ho et al., 2013). Neuroprotective effects of 6-shogaol were examined in a PD model, and it was observed that 6-shogaol protected against neuroinflammatory-induced damage of dopaminergic (DA) neurons via the inhibition of neuroinflammatory responses (Park et al., 2013). 1,8-cineole was seen to have neuroprotective effects against cortical neuronal/glial cell injury in in vitro model of ischaemia, and it was observed that the effects were due to its antioxidative properties (Ryu et al., 2014).

The targets identified in a previous study on network pharmacology of Withania somnifera (Duyu et al., 2021) against stress associated neurodegenerative disorders such as Alzheimer's and Parkinson's diseases were PTGS2, PTGS1, CHRM5, CHRNA4, CHRNA7, CHRNB2, ADORA1, KCNQ1, KCNQ2, PRKCD. These targets, PTGS2, PTGS1, CHRM5, CHRNA4, ADORA1 and PRKCD were present in the gene target list identified in this study. Similarly, another study that implemented network pharmacology focused only on Bacopa monnieri's action in neurological disorders (Jeyasri et al., 2020). It concluded with 52 of its phytoconstituents sharing interaction with 780 human targets. Some of the 780 human targets were seen to be present in this study's final list of 71 targets. For example, some common targets were PTPN1, STAT3, PTGS1, ADORA1, CHRM5, PRKCD, and PRKCA.

Withanolide $A$ is an essential phytoconstituent in this study. The compound-target network illustrates that Withanolide A shares connections with 24 gene targets, which in this study is the highest number of interactions a phytoconstituent has with the gene targets. The 24 targets include PRKCD, PTGS2, GSK3B and CASP8. The role of these targets in neurodegenerative diseases has been discussed. The 10 neurological disorders considered in this study share connections with one or more of these 24 targets. These gene targets are also present in the GO enriched terms such as serotonergic synapse, CAMP signalling pathway, neurotransmitter receptor activity, and chemical synaptic transmission. 
Out of the nine phytoconstituents, it is observed that Withanolide $A$ is mostly connected to those targets that share connections with many neurological disorders. This implies that Withanolide A can have a high impact on most of the mentioned neurological disorders. A similar analysis of the other phytoconstituents also revealed its relevance in neurological disorders.

It was observed that the bioactive scores of most of the phytoconstituents were almost in line with the various drugs prescribed for neurological disorders. Phytoconstituents like Withanolide A, 10-gingerol and Withaferin A have bioactivity comparable to Bromperidol, which is used to treat Dementia and Schizophrenia and Atomoxetine, which is prescribed to people who have ADHD. The probability of the compounds crossing the blood-brain barrier is $>78 \%$, and six of the nine phytoconstituents are in the $90 \%$ range, with the highest probability being $99 \%$.

The methodology followed in the current study is quite comprehensive, and specific selection criteria were followed to identify key ingredients, key phytoconstituents and crucial targets along with their multi-targeting and potential synergistic actions. Steps used in this study will be significantly helpful in analysing the role of phytoconstituents used in ayurvedic formulations in a more detailed and meaningful manner against complex neurological disorders.

\subsection{CONCLUSIONS}

The network pharmacological analysis carried out in this study supported that the five ingredients in SWRT may effectively prevent and treat neurological disorders. The screened targets were actively involved in the pathogenesis of neurological disorders. The Cytoscape networks unravelled strong links between the selected phytoconstituents and the targets. Some of the crucial targets identified were ALOX5, GSK3B, SLC9A1, CASP8, PTGS2, PRKCD, TRPV1, and nine phytoconstituents shared a connection to at least one or more of these targets. The PPI and enrichment analysis results had a strong neurological basis which provided more insight into the gene set considered in the study. The multitarget and multi-compound approach utilised throughout this study has opened up many options for further research, such as studying the dose-specific usage of this formulation as a whole versus using any of the ingredients independently or in combination.

Acknowledgements: There was no funding source for the research work.

Author Contributions: JC designed the project, supervised the work, analysed data and wrote the final manuscript; VJ , SVH \& KB designed and performed experiments, analysed the data and wrote the draft manuscript.

Conflicts of Interest: The authors declare no conflict of interest.

\section{References}

Abdul Manap, A. S., Vijayabalan, S., Madhavan, P., Chia, Y. Y., Arya, A., Wong, E. H., Rizwan, F., Bindal, U., \& Koshy, S. (2019). Bacopa monnieri, a Neuroprotective Lead in Alzheimer Disease: A Review on Its Properties, Mechanisms of Action, and Preclinical and Clinical Studies. Drug Target Insights, 13, 1177392819866412. https://doi.org/10.1177/1177392819866412

Abu-Taweel G. M. (2018). Cardamom (Elettaria cardamomum) perinatal exposure effects on the development, behavior and biochemical parameters in mice offspring. Saudi Journal of Biological Sciences, 25(1), 186-193. https://doi.org/10.1016/i.sjbs.2017.08.012

Aguiar, S., \& Borowski, T. (2013). Neuropharmacological review of the nootropic herb Bacopa monnieri. Rejuvenation Research, 16(4), 313-326. https://doi.org/10.1089/rej.2013.1431

Baitharu, I., Jain, V., Deep, S. N., Shroff, S., Sahu, J. K., Naik, P. K., \& Ilavazhagan, G. (2014). Withanolide A Prevents Neurodegeneration by Modulating Hippocampal Glutathione Biosynthesis during Hypoxia. PLoS ONE, 9(10), e105311. https://doi.org/10.1371/journal.pone.0105311

Batiha, G. E., Alkazmi, L. M., Wasef, L. G., Beshbishy, A. M., Nadwa, E. H., \& Rashwan, E. K. (2020). Syzygium aromaticum L. (Myrtaceae): Traditional uses, bioactive chemical constituents, pharmacological and toxicological activities. Biomolecules, 10(2), 202. https://doi.org/10.3390/biom10020202

Burguillos, M. A., Deierborg, T., Kavanagh, E., Persson, A., Hajji, N., Garcia-Quintanilla, A., Cano, J., Brundin, P., Englund, E., Venero, J. L., \& Joseph, B. (2011). Caspase signalling controls microglia activation and neurotoxicity. Nature, 472(7343), 319-324. https://doi.org/10.1038/nature09788

Chandran, U., Mehendale, N., Tillu, G., \& Patwardhan, B. (2015). Network Pharmacology: An Emerging Technique for Natural Product Drug Discovery and Scientific Research on Ayurveda. Proceedings of the Indian Academy of Sciences, 81, 8. https://doi.org/10.16943/ptinsa/2015/v81i3/48229 
Chaudhari, K. S., Tiwari, N. R., Tiwari, R. R., \& Sharma, R. S. (2017). Neurocognitive Effect of Nootropic Drug Brahmi (Bacopa monnieri) in Alzheimer's Disease. Annals of neurosciences, 24(2), 111-122. https://doi.org/10.1159/000475900

Chu, J., \& Praticò, D. (2011). 5-lipoxygenase as an endogenous modulator of amyloid $\beta$ formation in vivo. Annals of Neurology, 69(1), 34-46. https://doi.org/10.1002/ana.22234

Daina, A., Michielin, O., \& Zoete, V. (2019). SwissTargetPrediction: updated data and new features for efficient prediction of protein targets of small molecules. Nucleic Acids Research, 47(W1), W357-W364. https://doi.org/10.1093/nar/gkz382

Du, Y., Zhao, Y., Li, C., Zheng, Q., Tian, J., Li, Z., Huang, T. Y., Zhang, W., \& Xu, H. (2018). Inhibition of PKC $\delta$ reduces amyloid- $\beta$ levels and reverses Alzheimer disease phenotypes. Journal of Experimental Medicine, 215(6),1665-1677. https://doi.org/10.1084/jem.20171193

Dubey, K., Anand, B., Shekhawat, D., \& Kar, K. (2017). Eugenol prevents amyloid formation of proteins and inhibits amyloidinduced hemolysis. Scientific Reports, 7, 40744. https://doi.org/10.1038/srep40744

Duda, P., Wiśniewski, J., Wójtowicz, T., Wójcicka, O., Jaśkiewicz, M., Drulis-Fajdasz, D., Rakus, D., McCubrey, J. A., \& Gizak, A. (2018). Targeting GSK3 signaling as a potential therapy of neurodegenerative diseases and aging. Expert Opinion on Therapeutic targets, 22(10), 833-848. https://doi.org/10.1080/14728222.2018.1526925

Duyu, T., Khanal, P., Dey, Y.N., \& Jha, S. (2021).Network pharmacology of Withania somnifera against stress associated neurodegenerative diseases. Advances in Traditional Medicine, 21, 565-578. https://doi.org/10.1007/s13596-020$\underline{00530-x}$

Gadgil, V. D. (2010). Understanding ayurveda. Journal of Ayurveda and Integrative Medicine, 1(1), 77-80. https://doi.org/10.4103/0975-9476.59836

Galal, A. A. A., \& Abdellatief, S. A. (2015). Neuropharmacological studies on Syzygium aromaticum (clove) essential oil. International Journal of Pharma Sciences, 5(2): 1013-1018.

Gazova, Z., Soukup, O., Sepsova, V., Siposova, K., Drtinova, L., Jost, P., Spilovska, K., Korabecny, J., Nepovimova, E., Fedunova, D., Horak, M., Kaniakova, M., Wang, Z. J., Hamouda, A. K., \& Kuca, K. (2016). Multi-target-directed therapeutic potential of 7-methoxytacrine-adamantylamine heterodimers in the Alzheimer's disease treatment. Biochimica et Biophysica acta. Molecular Basis of Disease. 1863(2), 607-619. https://doi.org/10.1016/j.bbadis.2016.11.020

Gordon, R., Singh, N., Lawana, V., Ghosh, A., Harischandra, D. S., Jin, H., Hogan, C., Sarkar, S., Rokad, D., Panicker, N., Anantharam, V., Kanthasamy, A. G., \& Kanthasamy, A. (2016). Protein kinase C $\delta$ upregulation in microglia drives neuroinflammatory responses and dopaminergic neurodegeneration in experimental models of Parkinson's disease. Neurobiology of Disease, 93, 96-114. https://doi.org/10.1016/i.nbd.2016.04.008

Gorman A. M. (2008). Neuronal cell death in neurodegenerative diseases: recurring themes around protein handling. Journal of Cellular and Molecular Medicine, 12(6A), 2263-2280. https://doi.org/10.1111/j.1582-4934.2008.00402.x

Ha, S. K., Moon, E., Ju, M. S., Kim, D. H., Ryu, J. H., Oh, M. S., \& Kim, S. Y. (2012). 6-Shogaol, a ginger product, modulates neuroinflammation: a new approach to neuroprotection. Neuropharmacology, 63(2), 211-223. https://doi.org/10.1016/j.neuropharm.2012.03.016

Hamosh, A., Scott, A. F., Amberger, J. S., Bocchini, C. A., \& McKusick, V. A. (2005). Online Mendelian Inheritance in Man (OMIM), a knowledgebase of human genes and genetic disorders. Nucleic Acids Research, 33(Database issue), D514D517. https://doi.org/10.1093/nar/gki033

Ho, K. W., Ward, N. J., \& Calkins, D. J. (2012). TRPV1: a stress response protein in the central nervous system. American Journal of Neurodegenerative Disease, 1(1), 1-14.

Ho, S. C., Chang, K. S., \& Lin, C. C. (2013). Anti-neuroinflammatory capacity of fresh ginger is attributed mainly to 10gingerol. Food Chemistry, 141(3), 3183-3191. https://doi.org/10.1016/i.foodchem.2013.06.010

Iwama, K., Osaka, H., Ikeda, T., Mitsuhashi, S., Miyatake, S., Takata, A., Miyake, N., Ito, S., Mizuguchi, T., \& Matsumoto, N. (2018). A novel SLC9A1 mutation causes cerebellar ataxia. Journal of Human Genetics, 63(10), 1049-1054. https://doi.org/10.1038/s10038-018-0488-x

Jeyasri, R., Muthuramalingam, P., Suba, V., Ramesh, M., \& Chen, J. T. (2020). Bacopa monnieri and Their Bioactive Compounds Inferred Multi-Target Treatment Strategy for Neurological Diseases: A Cheminformatics and System Pharmacology Approach. Biomolecules, 10(4), 536. https://doi.org/10.3390/biom10040536

Kim, S., Chen, J., Cheng, T., Gindulyte, A., He, J., He, S., Li, Q., Shoemaker, B. A., Thiessen, P. A., Yu, B., Zaslavsky, L., Zhang, J., \& Bolton, E. E. (2021). PubChem in 2021: new data content and improved web interfaces. Nucleic Acids Research, 49(D1), D1388-D1395. https://doi.org/10.1093/nar/gkaa971

Kuhn, M., von Mering, C., Campillos, M., Jensen, L. J., \& Bork, P. (2008). STITCH: interaction networks of chemicals and proteins. Nucleic Acids Research, 36(Database issue), D684-D688. https://doi.org/10.1093/nar/gkm795

Kumar, A., Tiwari, A., \& Sharma, A. (2018). Changing paradigm from one target one ligand towards multi-target directed ligand design for key drug targets of Alzheimer disease: An important role of in silico methods in multi-target directed ligands design. Current Neuropharmacology, 16(6), 726-739. https://doi.org/10.2174/1570159X16666180315141643

Kumar, V., Dey, A., Hadimani, M.B., Marcovic, T., \& Emerald, M. (2015). Chemistry and pharmacology of Withania somnifera: An update. Tang, 5(1), 1.1-1.13. https://doi.org/10.5667/TANG.2014.0030 
Lei, P., Ayton, S., Bush, A. I., \& Adlard, P. A. (2011). GSK-3 in Neurodegenerative Diseases. International Journal of Alzheimer's Disease, 2011, 189246. https://doi.org/10.4061/2011/189246

Liu, Y., Liu, J., \& Zhang, Y. (2019). Research progress on chemical constituents of Zingiber officinale Roscoe. BioMed Research International, 2019, 5370823. https://doi.org/10.1155/2019/5370823

Mao, Q. Q., Xu, X. Y., Cao, S. Y., Gan, R. Y., Corke, H., Beta, T., \& Li, H. B. (2019). bioactive compounds and bioactivities of ginger (Zingiber officinale Roscoe). Foods, 8(6), 185. https://doi.org/10.3390/foods8060185

Mendez, D., Gaulton, A., Bento, A. P., Chambers, J., De Veij, M., Félix, E., Magariños, M. P., Mosquera, J. F., Mutowo, P., Nowotka, M., Gordillo-Marañón, M., Hunter, F., Junco, L., Mugumbate, G., Rodriguez-Lopez, M., Atkinson, F., Bosc, N., Radoux, C. J., Segura-Cabrera, A., Hersey, A., ... Leach, A. R. (2019). ChEMBL: towards direct deposition of bioassay data. Nucleic Acids Research, 47(D1), D930-D940. https://doi.org/10.1093/nar/gky1075

Parekar, R. R., Jadhav, K. S., Marathe, P. A., \& Rege, N. N. (2014). Effect of Saraswatarishta in animal models of behavior despair. Journal of Ayurveda and Integrative Medicine, 5(3), 141-147. https://doi.org/10.4103/0975-9476.140469

Park, G., Kim, H. G., Ju, M. S., Ha, S. K., Park, Y., Kim, S. Y., \& Oh, M. S. (2013). 6-Shogaol, an active compound of ginger, protects dopaminergic neurons in Parkinson's disease models via anti-neuroinflammation. Acta Pharmacologica Sinica, 34(9), 1131-1139. https://doi.org/10.1038/aps.2013.57

Piñero, J., Bravo, À., Queralt-Rosinach, N., Gutiérrez-Sacristán, A., Deu-Pons, J., Centeno, E., García-García, J., Sanz, F., \& Furlong, L. I. (2017). DisGeNET: a comprehensive platform integrating information on human disease-associated genes and variants. Nucleic Acids Research, 45(D1), D833-D839. https://doi.org/10.1093/nar/gkw943

Prabhu, J., Jayakumari, S., Prabhu, K., Kumar, J.A., Subramanian, M., \& Kavimani. (2019). Saraswatarishta reverses neuronal injury in brain tissues of scopolamine-induced rat model. Journal of Anatomical Society of India, 68(4), $269-273$. https:/doi.org/10.4103/JASI.JASI 4519

Prabhu, J., Prabhu, K., Chaudhuri, A., Rao, M. R. K., Kalai Selvi, V. S., Balaji, T. K., \& Dinakar, S. (2020). Neuro-protective effect of ayurveda formulation, saraswatharishtam, on scopolamine induced memory impairment in animal model. Pharmacognosy Journal, 12(1), 6-13. https://doi.org/10.5530/pj.2020.12.2

Rajopadhye, D. R., \& Sahasrabudhe, A. R. (2020). Memory enhancing activity of Saraswatarishta in mice. Biomedical and Pharmacology Journal, 13(4). https://dx.doi.org/10.13005/bpi/2082

Ram, N., Peak, S. L., Perez, A. R., \& Jinwal, U. K. (2021). Implications of Withaferin A in neurological disorders. Neural Regeneration Research, 16(2), 304-305. https://doi.org/10.4103/1673-5374.290894

Ramasamy, S., Chin, S. P., Sukumaran, S. D., Buckle, M. J. C., Kiew, L. V., \& Chung, L. Y. (2015) In silico and in vitro analysis of bacoside a aglycones and its derivatives as the constituents responsible for the cognitive effects of Bacopa monnieri. PLOS ONE, 10(5): e0126565. https://doi.org/10.1371/journal.pone.0126565

Raudvere, U., Kolberg, L., Kuzmin, I., Arak, T., Adler, P., Peterson, H., \& Vilo, J. (2019). g:Profiler: a web server for functional enrichment analysis and conversions of gene lists (2019 update). Nucleic Acids Research, 47(W1), W191-W198. https://doi.org/10.1093/nar/gkz369

Rawat, C., Kutum, R., Kukal, S., Srivastava, A., Dahiya, U. R., Kushwaha, S., Sharma, S., Dash, D., Saso, L., Srivastava, A. K., \& Kukreti, R. (2020). Downregulation of peripheral PTGS2/COX-2 in response to valproate treatment in patients with epilepsy. Scientific Reports, 10(1), 2546. https://doi.org/10.1038/s41598-020-59259-x

Ryu, S., Park, H., Seol, G. H., \& Choi, I. Y. (2014). 1,8-Cineole ameliorates oxygen-glucose deprivation/ reoxygenation-induced ischaemic injury by reducing oxidative stress in rat cortical neuron/glia. The Journal of Pharmacy and Pharmacology, 66(12), 1818-1826. https://doi.org/10.1111/jphp.12295

Safran, M., Solomon, I., Shmueli, O., Lapidot, M., Shen-Orr, S., Adato, A., Ben-Dor, U., Esterman, N., Rosen, N., Peter, I., Olender, T., Chalifa-Caspi, V., \& Lancet, D. (2002). GeneCards 2002: towards a complete, object-oriented, human gene compendium. Bioinformatics, 18(11), 1542-1543. https://doi.org/10.1093/bioinformatics/18.11.1542

Samad, T. A., Moore, K. A., Sapirstein, A., Billet, S., Allchorne, A., Poole, S., Bonventre, J. V., \& Woolf, C. J. (2001). Interleukin1 beta-mediated induction of Cox-2 in the CNS contributes to inflammatory pain hypersensitivity. Nature, 410(6827), 471-475. https://doi.org/10.1038/35068566

Sánchez, I., Xu, C. J., Juo, P., Kakizaka, A., Blenis, J., \& Yuan, J. (1999). Caspase-8 is required for cell death induced by expanded polyglutamine repeats. Neuron, 22(3), 623-633. https://doi.org/10.1016/s0896-6273(00)80716-3

Saroya A.S., \& Singh J. (2018) Neuropharmacology of Withania somnifera Dunal.. In: Pharmacotherapeutic Potential of Natural Products in Neurological Disorders. Springer, Singapore. https://doi.org/10.1007/978-981-13-0289-3 16

Shannon, P., Markiel, A., Ozier, O., Baliga, N. S., Wang, J. T., Ramage, D., Amin, N., Schwikowski, B., \& Ideker, T. (2003). Cytoscape: a software environment for integrated models of biomolecular interaction networks. Genome Research, 13(11), 2498-2504. https://doi.org/10.1101/gr.1239303

Shastri, RV., (Eds). (2002). Atha Vajikaranaprakaranam. Bhaisajyaratnavali, Vidyotini - Hindivyakhya - VimarshParishishtasahita. Varanasi: Chaukhamba Sanskrit Bhavan. 796-797.

Storozhuk, M. V., Moroz, O. F., \& Zholos, A. V. (2019). Multifunctional TRPV1 ion channels in physiology and pathology with focus on the brain, vasculature, and some visceral systems. BioMed Research International, 2019, 5806321. https://doi.org/10.1155/2019/5806321 
Szklarczyk, D., Gable, A. L., Lyon, D., Junge, A., Wyder, S., Huerta-Cepas, J., Simonovic, M., Doncheva, N. T., Morris, J. H., Bork, P., Jensen, L. J ., \& Christian, v. M. (2019). STRING v11: protein-protein association networks with increased coverage, supporting functional discovery in genome-wide experimental datasets. Nucleic Acids Research, 47(D1), D607D613. https://doi.org/10.1093/nar/gky1131

Viveros-Paredes, J. M., González-Castañeda, R. E., Gertsch, J., Chaparro-Huerta, V., López-Roa, R. I., Vázquez-Valls, E., BeasZarate, C., Camins-Espuny, A., \& Flores-Soto, M. E. (2017). Neuroprotective effects of $\beta$-caryophyllene against dopaminergic neuron injury in a murine model of Parkinson's disease induced by MPTP. Pharmaceuticals, $10(3), 60$. https://doi.org/10.3390/ph10030060

Yang, H., Lou, C., Sun, L., Li, J., Cai, Y., Wang, Z., Li, W., Liu, G., \& Tang, Y. (2019). admetSAR 2.0: web-service for prediction and optimisation of chemical ADMET properties. Bioinformatics, 35(6), 1067-1069. https://doi.org/10.1093/bioinformatics/bty707

Zheng, X. B., Zhang, Y. L., Li, Q., Liu, Y. G., Wang, X. D., Yang, B. L., Zhu, G. C., Zhou, C. F., Gao, Y., \& Liu, Z. X. (2019). Effects of 1,8-cineole on neuropathic pain mediated by P2X2 receptor in the spinal cord dorsal horn. Scientific Reports, $9,7909$. https://doi.org/10.1038/s41598-019-44282-4

Zhu, J., Park, S., Jeong, K. H., \& Kim, W. J. (2020). Withanolide-A treatment exerts a neuroprotective effect via inhibiting neuroinflammation in the hippocampus after pilocarpine-induced status epilepticus. Epilepsy Research, 165, 106394. https://doi.org/10.1016/j.eplepsyres.2020.106394 